\title{
THE HULL NUMBER OF AN ORIENTED GRAPH
}

\author{
GARY CHARTRAND, JOHN FREDERICK FINK, \\ and PING ZHANG
}

\author{
Received 27 October 2002
}

\begin{abstract}
We present characterizations of connected graphs $G$ of order $n \geq 2$ for which $h^{+}(G)=n$. It is shown that for every two integers $n$ and $m$ with $1 \leq n-1 \leq m \leq$ $\left(\begin{array}{l}n \\ 2\end{array}\right)$, there exists a connected graph $G$ of order $n$ and size $m$ such that for each integer $k$ with $2 \leq k \leq n$, there exists an orientation of $G$ with hull number $k$.
\end{abstract}

2000 Mathematics Subject Classification: 05C12, 05C20.

1. Introduction. The (directed) distance $d(u, v)$ from a vertex $u$ to a vertex $v$ in an oriented graph $D$ is the length of a shortest directed $u-v$ path in $D$. A directed $u-v$ path of length $d(u, v)$ is referred to as a $u-v$ geodesic. A vertex $w$ is said to lie in a $u-v$ geodesic $P$ if $w$ is an internal vertex of $P$, that is, $w$ is a vertex of $P$ distinct from $u$ and $v$. The closed interval $I[u, v]$ consists of $u$ and $v$ together with all vertices lying in a $u-v$ geodesic or in a $v-u$ geodesic in $D$. Hence, if there is neither a $u-v$ geodesic nor a $v-u$ geodesic in $D$, then $I[u, v]=\{u, v\}$. For a nonempty subset $S$ of $V(D)$, define

$$
I[S]=\bigcup_{u, v \in S} I[u, v] .
$$

Then certainly $S \subseteq I[S]$. A set $S$ is convex if $I[S]=S$. The convex hull $[S]$ of $S$ is the smallest convex set containing $S$. The set $[S]$ is also the intersection of all convex sets containing $S$. The convex hull $[S]$ of $S$ can also be formed from the sequence $\left\{I^{k}[S]\right\}, k \geq 0$, where $I^{0}[S]=S, I^{1}[S]=I[S]$, and $I^{k}[S]=I\left[I^{k-1}[S]\right]$ for $k \geq 2$. From some term on, this sequence must be constant. Let $p$ be the smallest number such that $I^{p}[S]=I^{p+1}[S]$. Then $I^{p}[S]$ is the convex hull $[S]$. A set $S$ of vertices of $D$ is called a hull set of $D$ if $[S]=V(D)$. A hull set of minimum cardinality is a minimum hull set of $D$. The cardinality of a minimum hull set in $D$ is called the hull number $h(D)$. Certainly, if $D$ is a nontrivial connected oriented graph of order $n$, then $2 \leq h(D) \leq n$.

Concepts related to hull sets and hull numbers in oriented graphs were studied in [7]. A set $S$ of vertices in an oriented graph $D$ is a geodetic set if $I[S]=V(D)$. A geodetic set of minimum cardinality is a minimum geodetic set, and this cardinality is the geodetic number $g(D)$. 


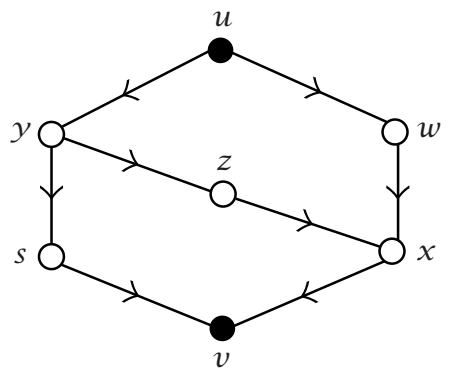

FigURE 1.1. An oriented graph $D$ with $h(D)=2$ and $g(D)=3$.

To illustrate these concepts, consider the oriented graph $D$ of Figure 1.1. Let $S=\{u, v\}$. Since $I[S]=V(D)-\{z\} \neq S$, it follows that $S$ is not convex. However, $[S]=I^{2}[S]=V(G)$ and so $S$ is a minimum hull set in $D$. Therefore, $h(D)=2$. On the other hand, the geodetic number of the oriented graph $D$ of Figure 1.1 is 3 and $\{u, v, z\}$ is a minimum geodetic set in $D$.

If $S$ is a hull set of an oriented graph $D$ and $u, v \in S$, then each vertex of every $u-v$ geodesic of $D$ belongs to $I[S]$. This observation implies the following lemma.

LEMMA 1.1. Let $S$ be a minimum hull set of an oriented graph $D$ and let $u, v \in S$. If $w$ lies in $a u-v$ geodesic in $D$, then $w \notin S$.

The degree $\operatorname{deg} v$ of a vertex $v$ in an oriented graph is the sum of its indegree and outdegree, that is, $\operatorname{deg} v=\operatorname{id} v+\operatorname{od} v$. A vertex $v$ is an endvertex if $\operatorname{deg} v=$ 1. A transmitter is a vertex having positive outdegree and indegree 0 , while a receiver is a vertex having positive indegree and outdegree 0 . For a vertex $u$ of $D$, let

$$
N^{+}(u)=\{x:(u, x) \in E(D)\}, \quad N^{-}(u)=\{x:(x, u) \in E(D)\} .
$$

So if $u$ is a transmitter, then $N^{-}(u)=\varnothing$; while if $v$ is a receiver, then $N^{+}(u)=$ $\varnothing$. A vertex $u$ of $D$ is a transitive vertex if (1) od $u>0$ and id $u>0$, (2) for every $v \in N^{+}(u)$ and $w \in N^{-}(u),(w, v) \in E(D)$. A vertex $v$ of $D$ is an extreme vertex if $v$ is a transmitter, receiver, or transitive vertex (see [2]). If $v$ is an extreme vertex, then $v$ can only be the initial or the terminal vertex of a geodesic containing $v$. This observation yields the following lemma.

LEMMA 1.2. Every hull set of a connected oriented graph D must contain the extreme vertices of $D$. In particular, every hull set of $D$ must contain its endvertices. Moreover, if the set of the extreme vertices of $D$ is a hull set, then it is the unique minimum hull set.

The closed intervals $I[u, v]$ in a connected graph were studied and characterized by Nebeský $[12,13]$ and were also investigated extensively in Mulder 


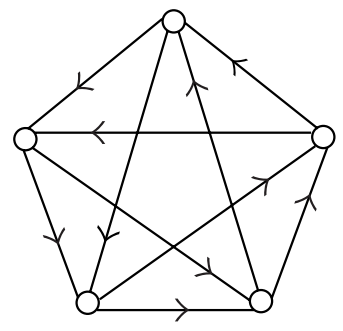

FIGURE 1.2. An oriented graph in which every pair of vertices is a hull set.

[10], where it was shown that these sets provide an important tool for studying metric properties of connected graphs. The sequential construction of a convex hull of a set of vertices in a graph was utilized in [9]. The hull number of a graph was introduced by Everett and Seidman [8] who characterized graphs having some particular hull numbers and who obtained a number of bounds for the hull numbers of graphs. The hull numbers of median graphs was determined by Mulder [11]. (A connected graph $G$ is a median graph if for every three vertices $u, v$, and $w$ of $G$, there is a unique vertex lying on a geodesic between each pair of $u, v$, and $w$.) The hull number of a graph was studied further in [3]. Convexity in graphs and digraphs was studied in [2, 6]. The geodetic number of a graph was introduced in [1] and studied further in [4], while the geodetic number of an oriented graph was studied in [7]. We refer to [1] for concepts and results on distance in graphs.

We have seen that if $D$ is a nontrivial connected oriented graph of order $n$, then

$$
2 \leq h(D) \leq n
$$

The upper and lower bounds in (1.3) are sharp for all $n \geq 2$. For example, the directed path $\overrightarrow{P_{n}}: v_{1}, v_{2}, \ldots, v_{n}$ of order $n \geq 2$ has hull number 2 , where the set $\left\{v_{1}, v_{n}\right\}$ is its unique minimum geodetic set. Obviously, the hull number of the directed cycle $\overrightarrow{C_{n}}$ is 2 as well, but in this case every pair of vertices in $\vec{C}_{n}$ is a hull set of $\overrightarrow{C_{n}}$. It was shown in [7] that $\overrightarrow{C_{n}}$ is the only connected oriented graph of order $n$ such that every pair of its vertices forms a geodetic set. However, this is not true for hull sets. It can be shown that every pair of vertices of the oriented graph in Figure 1.2 is a hull set.

At the other extreme are oriented graphs $D$ of order $n$ for which $h(D)=n$. We need an additional definition. An oriented graph $D$ is transitive if whenever $(u, v)$ and $(v, w)$ are $\operatorname{arcs}$ of $D$, then $(u, w)$ is an arc of $D$. We can now characterize oriented graphs of order $n$ having hull number $n$.

Proposition 1.3. Let $D$ be a nontrivial oriented graph of order $n$. Then $h(D)=n$ if and only if $D$ is transitive. 
Proof. Assume first that $h(D)<n$. Then there exists a vertex $v$ in $D$ such that $S=V(D)-\{v\}$ is a hull set of $D$. Then od $v>0$ and id $v>0$. This implies that $v$ lies in some $u-w$ geodesic $u, v, w$ in $D$, where $u, w \in S$. Therefore, $(u, w) \notin E(D)$ and $D$ is not transitive.

Conversely, assume that $D$ is an oriented graph that is not transitive. Then there exist distinct vertices $u, v$, and $w$ such that $(u, v),(v, w) \in E(D)$, but $(u, w) \notin E(D)$. Then $S=V(D)-\{v\}$ is a hull set and so $h(D)<n$.

Next we show that for a given integer $n \geq 2$, every integer $k$ with $2 \leq k \leq n$ is the hull number of some oriented graph of order $n$.

PROPOSITION 1.4. For every two integers $k$ and $n$ with $2 \leq k \leq n$, there exists an oriented graph of order $n$ and hull number $k$.

Proof. We show, in fact, that there exists an oriented graph with this property having the path $P_{n}$ of order $n$ as its underlying graph. Let $P_{n}: v_{1}, v_{2}, \ldots, v_{n}$. We construct an oriented graph $D$ from $P_{n}$ by directing the two edges incident with $v_{i}$ towards $v_{i}$ for all even $i$ with $i<k$. If $k$ is odd, then each edge $v_{i} v_{i+1}$ with $i \geq k$ is directed as $\left(v_{i+1}, v_{i}\right)$. If $k$ is even, then each edge $v_{i} v_{i+1}$ for $i \geq k-1$ is directed as $\left(v_{i}, v_{i+1}\right)$. In each case, the set $\left\{v_{1}, v_{2}, \ldots, v_{k-1}, v_{n}\right\}$ of extreme vertices is a hull set, so $h(D)=k$.

Next we provide an upper bound for the hull number of an oriented graph in terms of its order and diameter (the length of a longest geodesic).

PROPOSITION 1.5. If $D$ is a connected oriented graph of order $n$ and diameter $d$, then

$$
h(D) \leq n-d+1
$$

Proof. Let $u$ and $v$ be vertices of $D$ for which $d(u, v)=d$ and let $u=$ $v_{0}, v_{1}, \ldots, v_{d}=v$ be a $u-v$ geodesic. Let $S=V(D)-\left\{v_{1}, v_{2}, \ldots, v_{d-1}\right\}$. Then $[S]=V(D)$ and so $h(D) \leq|S|=n-d+1$.

Note that, in the proof of Proposition 1.4, the oriented graph $D$ constructed there has order $n$, diameter $d=n-k+1$, and hull number $k=n-d+1$. Therefore, the upper bound for $h(D)$ presented in Proposition 1.5 is sharp.

2. Relating hull number to geodetic number. If $D$ is a nontrivial connected oriented graph with $h(D)=a$ and $g(D)=b$, then necessarily $2 \leq a \leq b$. We now show that every pair $a, b$ of integers with $2 \leq a \leq b$ is realizable as the hull number and geodetic number, respectively, of some oriented graph. The following lemma is analogous to Lemma 1.2.

LEMMA 2.1. Every geodetic set of a connected oriented graph D must contain the extreme vertices of $D$. In particular, every geodetic set of D must contain its endvertices. Moreover, if the set of the extreme vertices of $D$ is a geodetic set, then it is the unique geodetic set. 


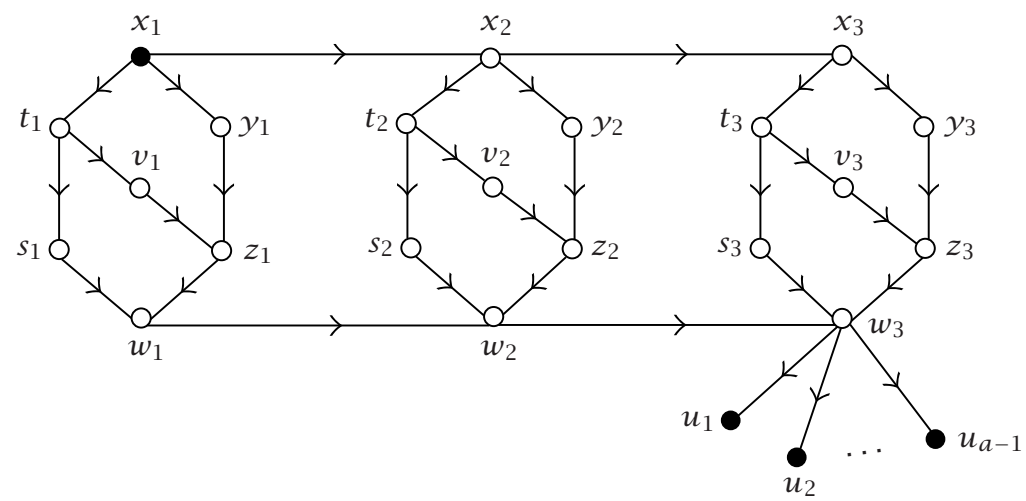

FIGURE 2.1. An oriented graph $D$ with $h(D)=a$ and $g(D)=b=a+3$.

THEOREM 2.2. For every pair $a, b$ of integers with $2 \leq a \leq b$, there exists $a$ connected oriented graph $D$ such that $h(D)=a$ and $g(D)=b$.

Proof. Assume first that $a=b \geq 2$. Let $D$ be the oriented graph whose underlying graph is the star $K_{1, a}$, where $V(D)=\left\{v, v_{1}, v_{2}, \ldots, v_{a}\right\}$ and $\operatorname{deg} v=$ $a$, and such that $\left(v_{1}, v\right) \in E(D)$ and $\left(v, v_{i}\right) \in E(D)$ for $2 \leq i \leq a$. Then $V(D)-$ $\{v\}$ is the set of extreme vertices of $D$. Since $V(D)-\{v\}$ is both a hull set and a geodetic set, it follows by Lemmas 1.2 and 2.1 that $h(D)=g(D)=\mid V(D)-$ $\{v\} \mid=a$.

Assume next that $2 \leq a<b$. We construct an oriented graph $D$ with the required hull and geodetic numbers. For each integer $i$ with $1 \leq i \leq b-a$, let $F_{i}$ be the oriented graph whose underlying graph is the 6-cycle $x_{i}, y_{i}, z_{i}, w_{i}$, $s_{i}, t_{i}, x_{i}$ and such that $F_{i}$ contains the two directed $x_{i}-w_{i}$ paths $x_{i}, y_{i}, z_{i}$, $w_{i}$ and $x_{i}, t_{i}, s_{i}, w_{i}$. The oriented graph $D_{i}$ is produced by adding the vertex $v_{i}$ and the two $\operatorname{arcs}\left(t_{i}, v_{i}\right)$ and $\left(v_{i}, z_{i}\right)$ to $F_{i}$. Therefore, $D_{i}$ is isomorphic to the oriented graph of Figure 1.1. The oriented graph $D$ is then obtained from the oriented graphs $D_{i}(1 \leq i \leq b-a)$ by adding (1) the $a-1$ new vertices $u_{j}(1 \leq j \leq a-1),(2)$ the $\operatorname{arcs}\left(w_{b-a}, u_{j}\right)$ for $1 \leq j \leq a-1$, and (3) the arcs $\left(x_{i}, x_{i+1}\right)$ and $\left(w_{i}, w_{i+1}\right)$ for $1 \leq i \leq b-a-1$. The oriented graph $D$ is shown in Figure 2.1 for $b-a=3$.

Let $U=\left\{u_{1}, u_{2}, \ldots, u_{a-1}\right\}$ and $V=\left\{v_{1}, v_{2}, \ldots, v_{b-a}\right\}$. Then $\left\{x_{1}\right\} \cup U$ is the set of extreme vertices of $D$. Since $I\left[\left\{x_{1}\right\} \cup U\right]=V(D)-V$ and $\left[\left\{x_{1}\right\} \cup U\right]=$ $I^{2}\left[\left\{x_{1}\right\} \cup U\right]=V(D)$, it follows that $\left\{x_{1}\right\} \cup U$ is a hull set of $D$ and so $h(G)=$ $\left|\left\{x_{1}\right\} \cup U\right|=a$ by Lemma 1.2.

Next we show that $g(G)=b$. Since $\left\{x_{1}\right\} \cup U \cup V$ is a geodetic set, $g(G) \leq$ $\left|\left\{x_{1}\right\} \cup U \cup V\right|=b$. It remains to show that $g(G) \geq b$. Let $W$ be a minimum geodetic set of $D$. Certainly, $\left\{x_{1}\right\} \cup U \subset W$ by Lemma 2.1. Of course, $W$ contains the vertex $x_{1}$ in $D_{1}$. We claim that $W$ contains at least one vertex in each 


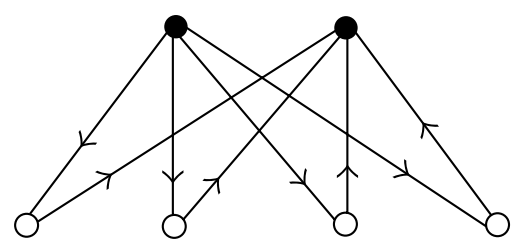

FIGURE 3.1. An oriented graph $D$ of $K_{2,4}$ with $h(D)=2$.

$D_{i}$ for all $i$ with $2 \leq i \leq b-a$. Otherwise, $V\left(D_{i}\right) \cap W=\varnothing$ for some $i$ with $2 \leq i \leq b-a$. Observe that $v_{i}$ does not lie on any $x-y$ geodesic in $G$ for $x, y \notin V\left(D_{i}\right)$. This implies that $v_{i} \notin I[W]$, which contradicts the fact that $W$ is a geodetic set. Therefore, as claimed, $W$ contains at least one vertex from each $D_{i}(2 \leq i \leq b-a)$ and so $|W| \geq\left|\left\{x_{1}\right\} \cup U\right|+(b-a-1)=b-1$. On the other hand, if $|W|=b-1$, then $W$ contains exactly one vertex from each $D_{i}$ $(1 \leq i \leq b-a)$. In particular, $x_{1}$ is the only vertex of $D_{1}$ belonging to $W$. Since each vertex $v_{i}(2 \leq i \leq b-a)$ only lies on those geodesics having $v_{i}$ as one of its endvertices or having both endvertices belonging to $D_{i}$, it follows that $v_{i} \in W$. This implies that $W=\left\{x_{1}\right\} \cup U \cup\left(V-\left\{v_{1}\right\}\right)$. However, then $v_{1} \notin I[W]$, which is a contradiction. Therefore, $g(D)=b$.

3. Orientable hull numbers of graphs. For a connected graph $G$ of order $n \geq 2$, the lower orientable hull number $h^{-}(G)$ of $G$ is defined as the minimum hull number among the orientations of $G$ and the upper orientable hull number $h^{+}(G)$ as the maximum hull number, that is,

$$
\begin{aligned}
& h^{-}(G)=\min \{h(D): D \text { is an orientation of } G\}, \\
& h^{+}(G)=\max \{h(D): D \text { is an orientation of } G\} .
\end{aligned}
$$

Hence, for every connected graph $G$ of order $n \geq 2$, we have $2 \leq h^{-}(G) \leq$ $h^{+}(G) \leq n$. First, we present a lemma that gives a sufficient condition for a graph to have lower hull number 2. Observe that if a graph $G$ contains a Hamiltonian path, then there exists an orientation $D$ of $G$ in which the Hamiltonian path in $G$ is oriented as a Hamiltonian path in $D$ such that $h(D)=2$. Thus we have the following lemma.

LEMMA 3.1. Let $G$ be a connected graph of order $n \geq 2$. If $G$ contains a Hamiltonian path, then $h^{-}(G)=2$.

The sufficient condition given in Lemma 3.1 for a graph to have lower orientable hull number 2 is not necessary. For example, the graph $K_{2,4}$ contains no Hamiltonian path. Since the orientation $D$ of $K_{2,4}$ of Figure 3.1 has $h(D)=2$, it follows that $h^{-}(G)=2$. 
Certainly, if $G$ contains a Hamiltonian path, then $G$ contains a spanning tree with two endvertices. More generally, we have the following lemma.

LEMMA 3.2. Let $\mu$ be the minimum number of endvertices among all spanning trees of a connected graph $G$. Then $h^{-}(G) \leq \mu$.

Proof. Let $T$ be a spanning tree of $G$ with $\mu$ endvertices, say, $v_{1}, v_{2}, \ldots, v_{\mu}$. We orient the edges of $T$ so that $T$ is rooted at $v_{1}$, that is, so that there is a directed path in $T$ from $v_{1}$ to every other vertex of $T$. For each edge $u v$ of $G$ not in $T$, if $d_{T}\left(v_{1}, u\right) \leq d_{T}\left(v_{1}, v\right)$, then orient $u v$ as $(v, u)$, and otherwise, orient $u v$ as $(u, v)$. We denote the resulting digraph by $D$.

Let $S=\left\{v_{1}, v_{2}, \ldots, v_{\mu}\right\}$. For distinct integers $i, j \in\{1,2, \ldots, \mu\}$, the only $v_{i}-$ $v_{j}$ geodesics in $D$ are the unique $v_{1}-v_{j}$ paths in $T$ with $2 \leq j \leq \mu$. Thus $I\left[v_{1}, v_{j}\right]$ contains all vertices of the unique $v_{1}-v_{j}$ path in $T$. Hence $V(D)=$ $\cup_{j=2}^{\mu} I\left[v_{1}, v_{j}\right] \subseteq I[S]$ and so $I[S]=V(D)$. Therefore, $h(D) \leq|S|=\mu$.

We now turn to those connected graphs $G$ of order $n \geq 2$ for which $h^{+}(G)=$ $n$. Recall that a vertex $v$ is an extreme vertex in an oriented graph if $v$ is a transmitter, a receiver, or a transitive vertex. Hence if there exists an orientation $D$ of a connected graph $G$ of order $n \geq 2$ such that every vertex of $D$ is an extreme vertex, then $h^{+}(G)=n$. The converse is also true for assume that $G$ is a connected graph of order $n \geq 2$ for which every orientation of $G$ has some vertex that is not an extreme vertex. Let $D$ be an orientation of $G$. Then $D$ contains a vertex $v$ that is not an extreme vertex. Hence $v$ is neither a transmitter, a receiver, nor a transitive vertex. Therefore, there exist two vertices $u$ and $w$ distinct from $v$ such that $(u, v)$ and $(v, w)$ are $\operatorname{arcs}$ of $D$ but $(u, w)$ is not an arc of $D$. Consequently, $v$ lies in a $u-w$ geodesic in $D$ and $V(D)-\{v\}$ is a hull set. Thus $h(D) \leq n-1$. Since $D$ is an arbitrary orientation of $G$, it follows that $h^{+}(G) \leq n-1$. We summarize these observations.

Proposition 3.3. Let $G$ be a connected graph of order $n \geq 2$. Then $h^{+}(G)=$ $n$ if and only if there exists an orientation $D$ of $G$ such that every vertex of $D$ is an extreme vertex.

For example, it is not difficult to show that there exists an orientation of any bipartite graph and any complete multipartite graph in which every vertex is an extreme vertex. Thus if $G$ is a bipartite graph or a complete multipartite graph of order $n \geq 2$, then $h^{+}(G)=n$.

There is yet another characterization of those connected graphs $G$ of order $n \geq 2$ for which $h^{+}(G)=n$. Let $S$ be a finite nonempty set of positive integers. The divisor graph $G(S)$ of $S$ has $S$ as its vertex set and two vertices $i$ and $j$ are adjacent if either $i \mid j$ or $j \mid i$. A graph $G$ is a divisor graph if $G=G(S)$ for some finite nonempty set $S$ of positive integers. The following theorem was proved in [5].

THEOREM 3.4. Let $G$ be a graph. Then $G$ is a divisor graph if and only if there exists an orientation $D$ of $G$ such that every vertex of $D$ is an extreme vertex. 
Combining Proposition 3.3 and Theorem 3.4, we have the following characterization which relates two concepts that, at the outset, would appear to be unrelated.

THEOREM 3.5. Let $G$ be a connected graph of order $n \geq 2$. Then $h^{+}(G)=n$ if and only if $G$ is a divisor graph.

There exist connected graphs $G$ and an integer $k$ with $h^{-}(G)<k<h^{+}(G)$ such that $G$ has no orientation with hull number $k$. In order to show this, we first determine the hull number of an orientation of a cycle.

Proposition 3.6. Let $D$ be an orientation of $C_{n}$ for $n \geq 3$. Then $h(D)=3$ or $h(D)=2 t$ for some integer $t$ with $1 \leq t \leq n / 2$.

Proof. Let $C_{n}: v_{1}, v_{2}, \ldots, v_{n}, v_{1}$ be a cycle of order $n$ and let $D$ be an orientation of $C_{n}$. Then the number $t$ of transmitters of $D$ equals the number of receivers of $D$. If $t=0$, then $D$ is a directed cycle and so $h(D)=2$. For $t \geq 1$, let $v_{i_{1}}, v_{i_{2}}, \ldots, v_{i_{t}}$ be the transmitters of $D$ and let $v_{j_{1}}, v_{j_{2}}, \ldots, v_{j_{t}}$ be the receivers of $D$, where $1 \leq i_{1}<i_{2}<\cdots<i_{t} \leq n$ and $1 \leq j_{1}<j_{2}<$ $\cdots<j_{t} \leq n$. By Lemma $1.2, h(D) \geq 2 t$. We assume, without loss of generality, that these transmitters and receivers appear around the cycle $C_{n}$ clockwise as $v_{i_{1}}, v_{j_{1}}, v_{i_{2}}, v_{j_{2}}, \ldots, v_{i_{t}}, v_{j_{t}}$.

CASE $1(t=1)$. If $d_{D}\left(v_{i_{1}}, v_{j_{1}}\right)=n / 2$, then $\left\{v_{i_{1}}, v_{j_{1}}\right\}$ is a hull set and so $h(D)=2$. If $d_{D}\left(v_{i_{1}}, v_{j_{1}}\right)<n / 2$, then $\left\{v_{i_{1}}, v_{j_{1}}\right\}$ is not a hull set and so $h(D) \geq 3$ by Lemma 1.2. On the other hand, $\left\{v_{i_{1}}, v_{i_{1}+1}, v_{j_{1}}\right\}$ or $\left\{v_{i_{1}-1}, v_{i_{1}}, v_{j_{1}}\right\}$ is a hull set. Thus $h(D)=3$.

CASE $2(t \geq 2)$. Since $\left\{v_{i_{1}}, v_{i_{2}}, \ldots, v_{i_{t}}, v_{j_{1}}, v_{j_{2}}, \ldots, v_{j_{t}}\right\}$ is a hull set of $D$, it follows by Lemma 1.2 that $h(D)=2 t$.

By Lemma 3.1 and Proposition 3.3, it is easy to verify that $(1) h^{-}\left(C_{n}\right)=2$ for all $n \geq 3$ and (2) $h^{+}\left(C_{n}\right)=n$ if $n=3$ or $n$ is even, while $h^{+}\left(C_{n}\right)=n-1$ if $n \geq 5$ is odd. Thus, by Proposition 3.6, if $n \geq 6$ and $5 \leq k<n$, where $k$ is odd, then there exists no orientation of $C_{n}$ with hull number $k$. Therefore, there are connected graphs $G$ such that $G$ has no orientation with hull number $k$ for some integer $k$ with $h^{-}(G)<k<h^{+}(G)$.

On the other hand, for every two integers $n$ and $m$ with $1 \leq n-1 \leq m \leq\left(\begin{array}{l}n \\ 2\end{array}\right)$, there exists a connected graph $G$ of order $n$ and size $m$ such that, for each integer $k$ with $2 \leq k \leq n$, there exists an orientation of $G$ with hull number $k$. In order to show this, we first present three lemmas. The converse $D^{*}$ of an oriented graph $D$ has the same vertex set as $D$ and the $\operatorname{arc}(u, v)$ in $D^{*}$ if and only if the arc $(v, u)$ is in $D$. Since the reversal of the edge directions on any $u-v$ geodesic in $D$ yields a $v-u$ geodesic in $D^{*}$ and vice versa, we have the following lemma.

LEMMA 3.7. If $D^{*}$ is the converse of an oriented graph $D$, then $h(D)=h\left(D^{*}\right)$.

LEMMA 3.8. Let $D$ be an oriented graph obtained from an oriented graph $D^{\prime}$ by adding a new vertex and joining it to all vertices of $D^{\prime}$. Then $h(D)=h\left(D^{\prime}\right)+1$. 
Proof. Let $v$ be the vertex of $D$ that is not in $D^{\prime}$. Since $\operatorname{id}_{D} v=0$, it follows that $v$ belongs to every hull set of $D$ by Lemma 1.2. Let $S^{\prime}$ be a minimum hull set of $D^{\prime}$. Then $\left[S^{\prime}\right]_{D^{\prime}}=V\left(D^{\prime}\right)$. Since $\left[S^{\prime} \cup\{v\}\right]=V\left(D^{\prime}\right) \cup\{v\}=V(D)$, it follows that $S \cup\{v\}$ is a hull set of $D$ and so $h(D) \leq\left|S^{\prime}\right|+1=h\left(D^{\prime}\right)+1$. On the other hand, let $S=A \cup\{v\}$ be a minimum hull set of $D$, where $A \subseteq V\left(D^{\prime}\right)$. Since every geodesic of $D$ is either a geodesic of $D^{\prime}$ or an $\operatorname{arc}\left(v, v^{\prime}\right)$ for some vertex $v^{\prime}$ in $D^{\prime}$, it follows that $V(D)=[S]=[A] \cup\{v\}$. Thus $[A]=V\left(D^{\prime}\right)$ and so $A$ is a hull set of $D^{\prime}$. So, $h\left(D^{\prime}\right) \leq|A|=|S|-1=h(D)-1$ or $h(D) \geq h\left(D^{\prime}\right)+1$. Therefore, $h(D)=h\left(D^{\prime}\right)+1$.

LEMMA 3.9. Let $D$ be an oriented graph obtained from an oriented graph $D^{\prime}$ by adding a new vertex $v$ and joining it to a vertex $v^{\prime}$ of $D^{\prime}$.

(a) If $v^{\prime}$ is a transmitter of $D^{\prime}$, then $h(D)=h\left(D^{\prime}\right)$.

(b) If $v^{\prime}$ is a receiver of $D^{\prime}$, then $h(D)=h\left(D^{\prime}\right)+1$.

Proof. First, assume that $v^{\prime}$ is a transmitter of $D^{\prime}$. Let $S^{\prime}$ be a minimum hull set of $D^{\prime}$. Then $v^{\prime} \in S^{\prime}$ and $\left[S^{\prime}\right]_{D^{\prime}}=V\left(D^{\prime}\right)$. Since every geodesic of $D$ is a geodesic in $D^{\prime}$ or a geodesic starting with $v$ followed by $v^{\prime}$, it follows that $\left(S^{\prime}-\left\{v^{\prime}\right\}\right) \cup\{v\}$ is a hull set of $D$. Thus $h(D) \leq\left|S^{\prime}\right|=h\left(D^{\prime}\right)$. On the other hand, let $S$ be a minimum hull set of $D$. Since $v$ is a transmitter of $D$, it follows by Lemma 1.2 that $v \in S$. Thus $S=\{v\} \cup A$, where $A \subseteq V\left(D^{\prime}\right)$. Since every $v-x$ geodesic of $D$, where $x \in V\left(D^{\prime}\right)$, has $v^{\prime}$ as its second vertex, it follows that $A \cup\left\{v^{\prime}\right\}$ is a hull set of $D^{\prime}$. Thus $h\left(D^{\prime}\right) \leq\left|A \cup\left\{v^{\prime}\right\}\right| \leq|A \cup\{v\}|=h(D)$. Therefore, $h(D)=h\left(D^{\prime}\right)$ and so (a) holds.

Next assume that $v^{\prime}$ is a receiver of $D^{\prime}$. Then $v^{\prime}$ is also a receiver of $D$ and so $v^{\prime}$ belongs to every hull set of $D$. Let $S^{\prime}$ be a minimum hull set of $D^{\prime}$. Then $v^{\prime} \in S^{\prime}$. Since $S^{\prime} \cup\{v\}$ is a hull set of $D$, it follows that $h(D) \leq\left|S^{\prime} \cup\{v\}\right|=$ $h\left(D^{\prime}\right)+1$. On the other hand, let $S$ be a minimum hull set of $D$. Since $v$ is a transmitter of $D$, it follows by Lemma 1.2 that $v \in S$. Thus $S=\{v\} \cup A$, where $A \subseteq V\left(D^{\prime}\right)$. Since $v$ is on no geodesic joining two vertices of $V\left(D^{\prime}\right)$, it follows that $A$ is a hull set of $D^{\prime}$. Thus $h\left(D^{\prime}\right) \leq|A| \leq h(D)-1$ or $h\left(D^{\prime}\right)+1 \leq h(D)$. Therefore, $h(D)=h\left(D^{\prime}\right)+1$ and so (b) holds.

We are now prepared to present the following result.

THEOREM 3.10. For every two integers $n$ and $m$ with $1 \leq n-1 \leq m \leq\left(\begin{array}{l}n \\ 2\end{array}\right)$, there exists a connected graph $G$ of order $n$ and size $m$ such that for each integer $k$ with $2 \leq k \leq n$ there exists an orientation of $G$ with hull number $k$.

PROOF. We prove the more general statement: for every two integers $n$ and $m$ with $1 \leq n-1 \leq m \leq\left(\begin{array}{l}n \\ 2\end{array}\right)$, there exists a connected graph $G$ of order $n$ and size $m$ having a vertex $v$ such that (a) $G$ contains a Hamiltonian path with initial vertex $v$ and (b) for each $3 \leq k \leq n$, there exists an orientation of $G$ having $v$ as a transmitter and hull number $k$. By Lemma 3.1, (a) and (b) imply that for each $2 \leq k \leq n$, there exists an orientation of $G$ having $v$ as a transmitter and hull number $k$. 
We proceed by induction on $n$. Since the statement is certainly true if $n \leq 3$, we assume that $n \geq 4$. Suppose that the statement is true for $n-1$. We consider two cases.

CASE $1\left(2 n-3 \leq m \leq\left(\begin{array}{l}n \\ 2\end{array}\right)\right)$. Then $(n-1)-1 \leq m-(n-1) \leq\left(\begin{array}{c}n-1 \\ 2\end{array}\right)$. By the induction hypothesis, there exists a connected graph $G^{\prime}$ of order $n-1$ and size $m-(n-1)$ having a vertex $v^{\prime}$ such that $G^{\prime}$ contains a Hamiltonian path starting at $v^{\prime}$ and for each $3 \leq k \leq n-1$, there exists an orientation of $G^{\prime}$ having $v^{\prime}$ as a transmitter and hull number $k$.

Let $G=G^{\prime}+K_{1}$ with $V\left(K_{1}\right)=\{v\}$. Then $G$ is a connected graph of order $n$ and size $m$. Since $G^{\prime}$ contains a Hamiltonian path starting at $v^{\prime}$, it follows that $G$ contains a Hamiltonian path starting at $v$. Thus $G$ has an orientation with hull number 2 by Lemma 3.1. We now assume that $3 \leq k \leq n$. Then $2 \leq k-1 \leq n-1$. By the induction hypothesis, there exists an orientation $D^{\prime}$ of $G^{\prime}$ having $v^{\prime}$ as a transmitter and hull number $k-1$. We extend the orientation $D^{\prime}$ of $G^{\prime}$ to an orientation $D$ of $G$ by directing each edge incident with $v$ in $G$ away from $v$. Then $v$ is a transmitter of $D$. Since $h\left(D^{\prime}\right)=k-1$, it then follows by Lemma 3.8 that $h(D)=k$.

CASE $2(n-1 \leq m \leq 2 n-4)$. Then $(n-1)-1 \leq m-1 \leq 2 n-5$. By the induction hypothesis, there exists a connected graph $G^{\prime}$ of order $n-1$ and size $m-1$ having a vertex $v^{\prime}$ such that $G^{\prime}$ contains a Hamiltonian path starting at $v^{\prime}$ and for each $3 \leq k \leq n-1, G^{\prime}$ has an orientation having $v^{\prime}$ as a transmitter and hull number $k$. Let $G$ be the graph obtained from $G^{\prime}$ by adding a new vertex $v$ and the pendant edge $v v^{\prime}$. Then $G$ has order $n$ and size $m$. Since $G^{\prime}$ contains a Hamiltonian path starting at $v^{\prime}$, it follows that $G$ contains a Hamiltonian path starting at $v$. So, $G$ has an orientation with hull number 2 by Lemma 3.1. For $3 \leq k \leq n$, we consider two subcases.

SuBCASE $2.1(3 \leq k \leq n-1)$. Let $D^{\prime}$ be an orientation of $G^{\prime}$ having $v^{\prime}$ as a transmitter and $h\left(D^{\prime}\right)=k$. We extend $D^{\prime}$ to an orientation of $G$ by directing the edge $v v^{\prime}$ as $\left(v, v^{\prime}\right)$. Then $v$ is a transmitter of $D$. By Lemma 3.9, $h(D)=$ $h\left(D^{\prime}\right)=k$.

SUBCASE $2.2(k=n)$. Let $D^{\prime}$ be an orientation of $G^{\prime}$ having $v^{\prime}$ as a transmitter and $h\left(D^{\prime}\right)=n-1$ and let $D^{*}$ be the converse of $D^{\prime}$. Then $v^{\prime}$ is a receiver of $D^{*}$ and $h\left(D^{*}\right)=h\left(D^{\prime}\right)=n-1$ by Lemma 3.7. We now extend $D^{*}$ to an orientation $D$ of $G$ by directing the edge $v v^{\prime}$ as $\left(v, v^{\prime}\right)$. Then $v$ is a transmitter of $D$. It then follows by Lemma 3.9 that $h(D)=h\left(D^{*}\right)+1=n$.

There is reason to believe that if $G$ is any connected graph of order $n \geq 3$, then $h^{-}(G) \neq h^{+}(G)$. Consequently, we conclude this paper with the following conjecture.

CONJECTURE 3.11. For every connected graph $G$ of order at least $3, h^{-}(G) \neq$ $h^{+}(G)$.

ACKNOWLedgment. This research was supported in part by the Western Michigan University Research Development Award Program. 


\section{REFERENCES}

[1] F. Buckley and F. Harary, Distance in Graphs, Addison-Wesley Publishing, California, 1990.

[2] G. Chartrand, J. F. Fink, and P. Zhang, Convexity in oriented graphs, Discrete Appl. Math. 116 (2002), no. 1-2, 115-126.

[3] G. Chartrand, F. Harary, and P. Zhang, On the hull number of a graph, Ars Combin. 57 (2000), 129-138.

[4] _ On the geodetic number of a graph, Networks 39 (2002), no. 1, 1-6.

[5] G. Chartrand, R. Muntean, V. Saenpholphat, and P. Zhang, Which graphs are divisor graphs? Congr. Numer. 151 (2001), 189-200.

[6] G. Chartrand, C. E. Wall, and P. Zhang, The convexity number of a graph, Graphs Combin. 18 (2002), no. 2, 209-217.

[7] G. Chartrand and P. Zhang, The geodetic number of an oriented graph, European J. Combin. 21 (2000), no. 2, 181-189.

[8] M. G. Everett and S. B. Seidman, The hull number of a graph, Discrete Math. 57 (1985), no. 3, 217-223.

[9] F. Harary and J. Nieminen, Convexity in graphs, J. Differential Geom. 16 (1981), no. $2,185-190$.

[10] H. M. Mulder, The Interval Function of a Graph, Mathematical Centre Tracts, vol. 132, Mathematisch Centrum, Amsterdam, 1980.

[11]__ The expansion procedure for graphs, Contemporary Methods in Graph Theory (R. Bodendiek, ed.), Bibliographisches Institut, Mannheim, 1990, pp. 459-477.

[12] L. Nebeský, A characterization of the interval function of a connected graph, Czechoslovak Math. J. 44(119) (1994), no. 1, 173-178.

[13] Characterizing the interval function of a connected graph, Math. Bohem. 123 (1998), no. 2, 137-144.

Gary Chartrand: Department of Mathematics, Western Michigan University, Kalamazoo, MI 49008, USA

E-mail address: chartrand@wmich.edu

John Frederick Fink: Department of Mathematics, The University of Michigan-Dearborn, Dearborn, MI 48128-1491, USA

E-mail address: jffink@umich.edu

Ping Zhang: Department of Mathematics, Western Michigan University, Kalamazoo, MI 49008, USA

E-mail address: ping.zhang@wmich.edu 


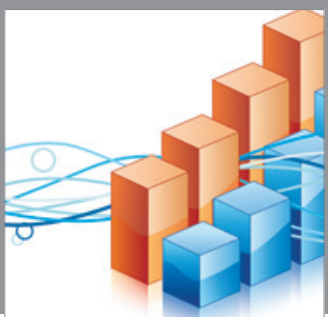

Advances in

Operations Research

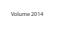

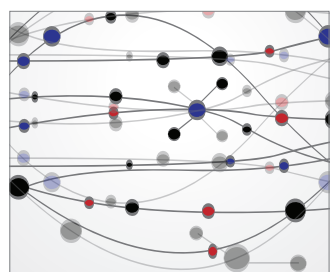

\section{The Scientific} World Journal
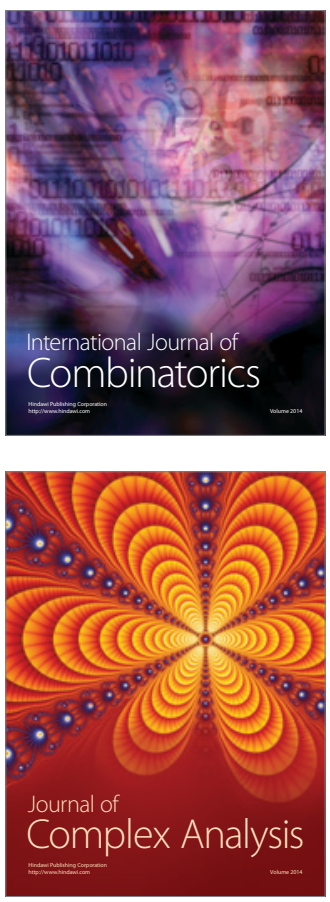

International Journal of

Mathematics and

Mathematical

Sciences
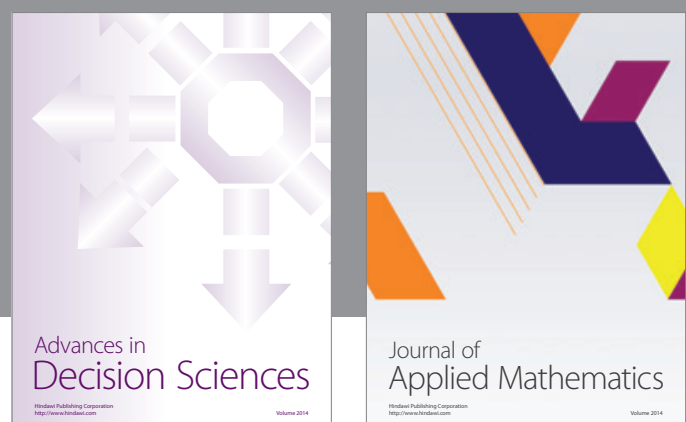

Journal of

Applied Mathematics
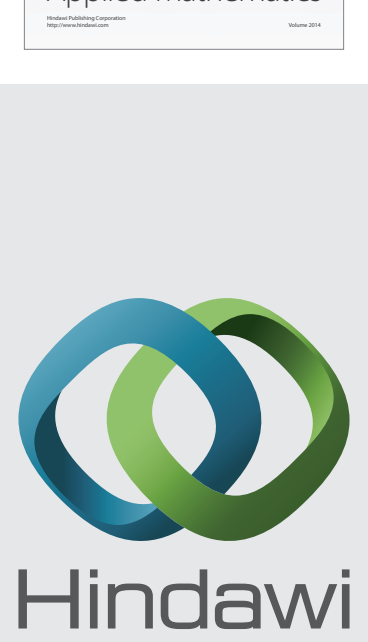

Submit your manuscripts at http://www.hindawi.com
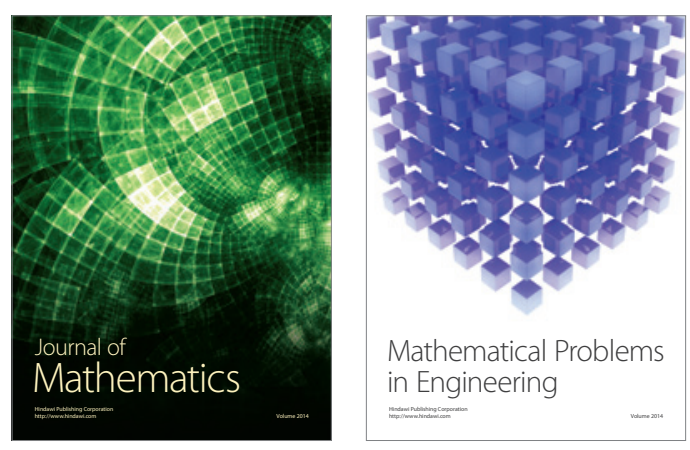

Mathematical Problems in Engineering
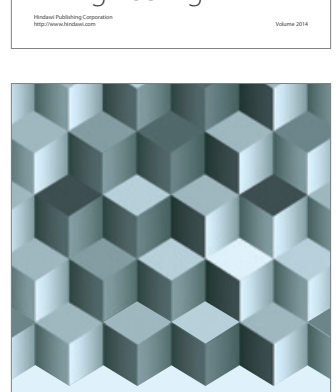

Journal of

Function Spaces
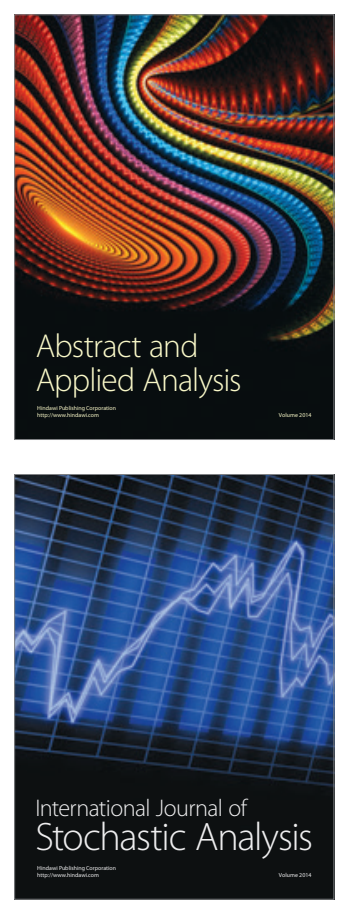

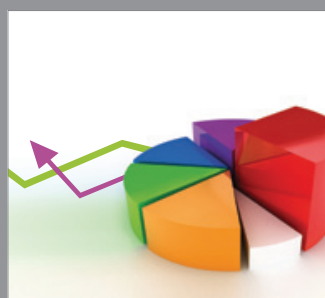

ournal of

Probability and Statistics

Promensencen
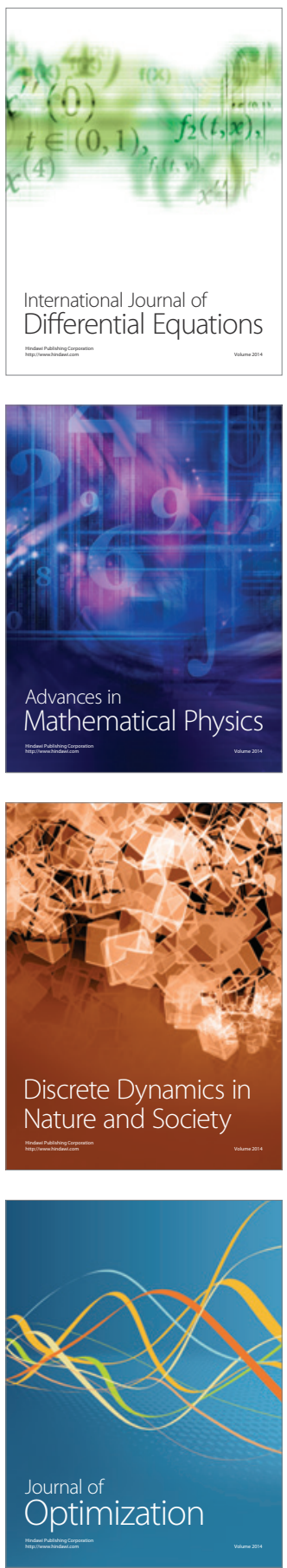